\title{
REIMAGINAR LA CIUDAD. PRÁCTICAS \\ DE OCIO JUVENIL Y PRODUCCIÓN DEL ESPACIO PÚBLICO URBANO ${ }^{1}$

\author{
REIMAGINING THE CITY. YOUTH LEISURE PRACTICES
} AND THE PRODUCTION OF PUBLIC URBAN SPACE
}

\author{
Fernando Bayón \\ Instituto de Estudios de Ocio \\ Facultad de Ciencias Sociales y Humanas \\ Universidad de Deusto, España \\ fernandobayon@deusto.es \\ Jaime Cuenca \\ Instituto de Estudios de Ocio \\ Facultad de Ciencias Sociales y Humanas \\ Universidad de Deusto, España \\ jaime.cuenca@deusto.es \\ José Antonio Caride \\ Facultad de Ciencias de la Educación \\ Universidad de Santiago de Compostela, España \\ joseantonio.caride@usc.es
}

Como citar / Citation

Bayón, Fernando; Cuenca, Jaime y Caride, José Antonio (2017) "Reimaginar

la ciudad. Prácticas de ocio juvenil y producción del espacio público urba-

\footnotetext{
${ }^{1}$ El trabajo que se presenta toma como referencia dos proyectos de investigación que, en el marco de dos convocatorias del Plan Nacional de I+D+i, han sido financiados por el Ministerio de Economía y Competitividad del Gobierno de España y por los Fondos FEDER de la Unión Europea: "De los tiempos educativos a los tiempos sociales: la construcción cotidiana de la condición juvenil en una sociedad de redes. Problemáticas específicas y alternativas pedagógico-sociales" (EDU2012-39080-C07-01 a 07_RESORTES) y "Educar el ocio: Realidades y perspectivas en clave intergeneracional, integral e inclusiva en una sociedad de redes" (EDU2015-65638-C6-1-R_ENREDOS).
} 
no". OBETS. Revista de Ciencias Sociales, 12(Extra 1): pp-pp. 21-41

doi:10.14198/OBETS2017.12.1.10

\section{Resumen}

Tras recorrer algunas de las recientes transformaciones discursivas en la comprensión del espacio urbano, este artículo teórico analiza tres prácticas de ocio juvenil, seleccionadas por su especial imbricación con la trama de la ciudad: el skateboarding, el graffiti y el juego de realidad aumentada Pokémon Go. Se pretende así contribuir a la comprensión del papel de las formas contemporáneas del ocio juvenil, y los tipos de experiencia territorial que les son propias, en la reestructuración del imaginario urbano y la producción del espacio público en la actualidad.

Palabras clave: ocio juvenil; espacio público; ciudad; imaginario.

\section{Abstract}

After reviewing some of the most recent discursive transformations in the understanding of urban space, this theoretical article analyses three youth leisure practices, which were selected because of their special link with the city plot: skateboarding, graffiti and the augmented reality game Pokémon Go. We intend to contribute to the understanding of the role of contemporary forms of youth leisure, and the their own types of experience, in the restructuring of the urban imaginary and the production of the public space nowadays.

Keywords: youth leisure; public space; city; imaginary.

\section{Extended abstract}

One of the most renowned analyses of critical urban geography, that of Henri Lefebvre (2013), assigned three specific characteristics to the space of the modern city: homogeneity, fragmentation and hierarchy. Even if his fundamental hypothesis, concerning the nature of space as a social product, remains unchallenged -or precisely because of that-, Lefebvre's description of urban space cannot simply continued to be applied to contemporary cities as it was when it was formulated back in the $60 \mathrm{~s}$ and $70 \mathrm{~s}$. The fabric and structure of modern urbanism was being contested then on the name of creativity and imagination by the Situationist International. But contemporary performative critiques to the ruling spatial principles of urban life need to come from different places and follow different strategies if the principles themselves have been thoroughly transformed. The strictly structured and rationalized city of Lefebvre has been replaced by the evanescent, flux-centered and -as suggested by Sloterdijk (2014)- foam-resembling postmetropolis of today.

One of the prevalent metaphors among urban development decision-makers and city branding managers is that of the city as an ever-changing scene always able to stage new and exciting events. In this light, leisure is not any more a free-time phenomenon giving functional meaning to clearly delimited areas of the urban plot. Rather, as a privileged arena for the late modern quest for intense lived experiences, leisure becomes a driving force in bringing forth new ways of understanding and living the city through embodied and socially meaningful action. This theoretical article intends to shed light on the role three 
specific youth leisure practices play in restructuring urban imaginaries and producing the public space of today: street skateboarding, graffiti and the augmented reality game Pokémon Go. Very different from each other in their history, the resources they require and the skills they help to develop, these three leisure practices though have something in common: they represent emplaced types of action that enact a creative and playful experience of the city. The selected youth leisure practices are analyzed in the article taking into account their own internal social logic and the language with which they imagine and appropriate public space. There are three main questions being addressed by our interpretation of the most relevant current scientific literature on these practices: first, how do they interact with urban space, understood as a social product; second, how do their participants re-imagine and re-signify the public space in the city; and third, how do they help define new functions and opportunities for the territory of the contemporary city, often encountering a hostile reaction while doing so.

Born as a youth leisure practice in southern California in the 50s, skateboarding was first located in found spaces (such as empty pools) and, later, in ad hoc built skateparks. It was only in the 80s that skaters began to choose downtown locations and use urban furniture for their 'tricks', hence marking the birth of street skateboarding. It encountered a degree of hostility among the general public not matched by other outdoor urban sport activities, which most probably relies on its condition of unstructured expressive physical activity, not linked to an external reward (Beal \& Weidman, 2003). Thanks to this peculiar type of activity, skaters develop their own imaginary around and a specific experience of the urban environment they use as a playground, thus embodying a performative critique of the city (Borden, 1998a, 2001a, 2001b; Woolley \& Johns, 2001).

The contemporary graffiti scene first developed in New York in the 70s, along other expressions of Afro-American hip hop youth culture, such as rap or breakdance. What remains specific about graffiti, as a visual creative practice, is that it cannot be understood without taking seriously its territorial condition. In order to achieve the sought after reputation, any graffiti writer (as graffiti artists are known) needs to balance the display of aesthetic skills with an extensive presence -mainly of his or her signature (tag)- throughout the city. Hence writers imagine the city as a space full of possibilities to assure peer recognition through an exercise of creative self-expression: the city is understood, not as a homogeneous unit, but as a continuous plane of multiple surfaces and interfaces (Halsey \& Young, 2006). Such a comprehension of urban space implies a neutralization of the marks and signs that intend to order it, a performative critique of officially sanctioned hierarchies of use and function, which explains the crusades against graffiti so many city councils around the globe have embarked on.

In July 2016, the American software development company Niantic launched Pokémon Go, an augmented reality location based game for iOS and Android mobile devices. Pokémon Go was one of the most popular apps of 2016 worldwide and by February 2017 its number of downloads amounted to 650 million, which makes it one of the youth leisure practices of greatest 
global impact in the last years. The hybrid nature of the game, consisting of the superimposition of physical and digital environments, allows the player to enjoy a specific experience of urban space: by adding digital elements, trivial and familiar locations become meaningful game loci, scenes of a ludic adventure through the city (Hjorth y Richardson, 2017). This combination of familiarity and excitement has a powerful effect on the players, many of whom change their daily routines because of the game, engaging in behaviour easily ridiculed by the media.

After analysing the three selected forms of contemporary youth leisure some commonalities become apparent. In their practice, the skater, the graffiti writer and the Pokémon Go player gain access to very specific experiences of urban environment and enact a lived space that is beyond some of the most fundamental features of modern urbanism, such as functional zoning and strictly delimited forms of land ownership. This kind of space is not totally unrelated to the most prevalent metaphors of the contemporary city; only, it does not rise from the abstract field of city branding, but from embodied and emplaced action in the living urban plot. That is what makes this three leisure practices plausible strategies to retake and reimagine public space in late modern cities.

\section{INTRODUCCIÓN}

La convicción de que se puede escrutar la génesis de la sociedad actual a través del espacio producido, se remonta al menos a los trabajos de Henri Lefebvre. En su ensayo filosóficamente más ambicioso, La producción del espacio, dejaba constancia de que el espacio de la modernidad posee tres caracteres precisos, que inicialmente pueden parecer incluso antagónicos: homogeneidad, fragmentación y jerarquización. Los modelos de gestión, así como las exigencias de vigilancia y comunicación, del espacio tienden a volverlo homogéneo en todas sus partes. Sin embargo, este espacio homogéneo se fragmenta en lotes, se deshilvana en bloques o parcelas, que acaban produciendo clausuras y guetos, pseudo-conjuntos pobremente vinculados con los centros urbanos. Además, esta fragmentación obedece a una estricta jerarquización: espacios residenciales, espacios comerciales, espacios de ocio, espacios marginales, etc. (Lefebvre, 2013: 58)

Este esquema del espacio moderno, cuyas raíces se hunden al menos en el París de Napoléon III, pero cuya paternidad más reciente suele atribuirse comúnmente a quienes, como la escuela Bauhaus o Le Corbusier, fueron primero tildados de revolucionarios para acabar posteriormente adoptados por el capitalismo avanzado, con su lógica de homogeneidad-fragmentación-jerarquización, puede que dé muestras de fatiga si recurrimos a él para explicar el comportamiento del espacio social contemporáneo. Pese a todo, la caracteriza- 
ción de Lefebvre sigue vigente en muchos de sus puntos clave: la homogeneidad del espacio oculta los conflictos reales, situación que el sociólogo francés cree extensible al funcionamiento del saber y la cultura, y aun de toda la sociedad, tras la Modernidad. Su hipótesis fundamental, la concepción del espacio como producto social, choca todavía hoy contra dos ilusiones. La primera es la ilusión de la transparencia, según la cual el espacio aparece como algo inteligible, un campo libre a la acción. Como alguien que circula cotidianamente por una región que disimula perfectamente sus peligros y oculta sus trampas, el ciudadano no ve detrás del diseño ningún designio. La segunda es la ilusión realista, que tiende a naturalizar ese artificio social llamado espacio urbano. La masiva aceptación de la facticidad del espacio, como un dato que se da por sentado dentro de lo que Blumenberg denominara "el mundo de la vida" (Blumenberg, 1999: 51), parece dejar claro hasta qué punto ha triunfado el rasgo general de la producción en el capitalismo, subrayado por la tradición marxista que va de Walter Benjamin a Henri Lefebvre: en los productos, sea un objeto o un espacio -o un espacio como objeto- se borran las huellas de la actividad productora tanto como es posible (Lefebvre, 2013: 255).

La ley del espacio urbano de la modernidad, tan poderosamente escrutada por Lefebvre, conoce sin embargo en nuestros días adendas múltiples e impugnaciones profundas. La organización del espacio público ha estado inspirada tradicionalmente por una cierta antropología social que repartía la urbe en zonas de accesibilidad normal, zonas de frontera -que imponían vedas relativas o absolutas a los flujos-, zonas residenciales y puntos de sutura que servían principalmente como lugares de encuentro e intercambio. Esta distribución ha padecido todo tipo de reformulaciones a lo largo de la historia; pero, con todo, ha mantenido su funcionalidad y, lo que acaso haya resultado más importante, ha normalizado un tipo de "civilidad" cuyo principal blasón ético era el de saber estar en el momento preciso en el espacio oportuno haciendo algo útil o aceptable que consintiera con la razón de ser del lugar.

Esta disciplina de producción del espacio urbano generó muy pronto contestaciones de diversa naturaleza, que coincidían en la pretensión de evidenciar el carácter restrictivo y anti-imaginativo de la ciudad burguesa del capitalismo avanzado. Seguramente una de las contestaciones más subversivas en ese sentido fue la protagonizada por la Internacional Situacionista que liderara a mediados de los años cincuenta Guy Debord quien, tanto en su Introducción a una crítica de la geografía urbana como especialmente en Teoría de la deriva, defiende un modo de comportamiento experimental ligado a las condiciones de la sociedad urbana, inspirado en técnicas de paso fugaz a través de ambientes aleatorios y heterogéneos, cuyos antecedentes habría que buscarlos en el 
surrealismo (Fargue: 2014; Aragon: 2016), inspirador también del flâneur benjaminiano (Benjamin, 2005). La ciudad ya no es un damero sobre el que deslizarnos ordenadamente, igual que las fichas de un juego regulado preliminarmente por agencias de autoridad indiscutida, sino un espacio discontinuo de puntos y vórtices que constituye una cartografía modulada por el estado de conciencia de cada uno de los que penetran en ella mezclando su exposición a los designios del azar con la intensificación de comportamientos afectivos hacia el medio físico (AAVV, 2006; Andreotti y Costa, 1996; Careri, 2013: 78-79). Pues las ciudades han transformado la anatomía de sus espacios de modo que, como afirma Peter Sloterdijk en el cierre de su trilogía "Esferas":

Se definen, por una parte, como emplazamientos de colectores pensados para colectivos reunibles; alojan, por otra, los complejos de apartamentos que sirven de cápsulas-vivienda a familias pequeñas o a quienes viven solos; $y$, finalmente, albergan las numerosas instalaciones del mundo del trabajo, en las que la mayoría de los habitantes de las ciudades aseguran sus bases económicas de existencia. Para la tarea de conformar un espacio común sobre los tres polos de la vida ciudadana (trabajo, vivienda, espacio público y colector) se han impuesto en la literatura urbanística las expresiones de tráfico y comunicación; como si se quisiera reducir el fenómeno ciudad a las generalidades del cambio de lugar y del flujo de datos. Desde que el impulso electrónico ha alcanzado a la teoría, esto llega hasta ficciones como la de la ciudad virtual, el territorio-online, la City of Bits, la Ciberville y metáforas de descorporeización semejantes. (Sloterdijk, 2014: 497)

Estamos, por lo tanto, ante un urbanismo-e que parece dejar muy atrás el concepto de "deriva" de Guy Debord: cuando el modelo de urbanismo vigente vaporiza la ciudad contemporánea en nombre de un nuevo romanticismo de la inmaterialidad que volatiliza las viejas zonificaciones de la cartografía urbana tardoburguesa (trabajo/residencia/ocio), y sustituye las localizaciones físicas por tramas casi fantasmales de nodos telemáticos (disembedding), todas las modalidades del flâneur y los mitos liberadores asociados al vagabundeo y la errancia parecen de repente trasnochados esfuerzos por reventar los límites físicos de lo que no cabe ya imaginar sino como una macro-espuma. Y precisamente estos cambiantes modos de imaginar la ciudad deben escrutarse con la mayor atención, porque el espacio no es algo dado, sino algo imaginado.

Asistimos, como habitantes de las ciudades, a una lucha diaria por la territorialización del espacio público urbano. Las modernas urbes apuestan cada vez más por la versatilidad de sus espacios, en la confianza de que, de este modo, sus calles y plazas puedan parecerse idealmente a un escenario acogedor de eventos múltiples. El espacio público urbano tiene que acreditar, como toda la fuerza de trabajo del capitalismo de mercado, su perenne y atlética disponi- 
bilidad a ser usada. Estas políticas mercadotécnicas, urgidas por el llamado city-branding que, según se insiste, "pone a las ciudades en el mapa", parecen apoyarse en el éxito de la metáfora del escenario, olvidando -o no- que el escenarium era la plataforma elevada dentro de un teatro que se reservaba para las representaciones... y de la que quedaba excluido, por definición, el público (Bayón, 2012: 410). En esta conversión de la ciudad en escenario de todo tipo de eventos (deportivos, culturales y lúdicos) desempeña un papel crucial el amplio repertorio de ofertas de ocio de las sociedades de consumo contemporáneas. El ocio no representa ya hoy, como en el urbanismo moderno, un conjunto de ocupaciones del tiempo libre al que se consagran funcionalmente ciertas áreas que, junto a las residenciales y las del trabajo, estructuran la trama urbana en una tríada de tanques estancos. Al contrario, como cauce privilegiado para canalizar la constante exigencia tardomoderna de vivencias excitantes en lo cotidiano (Cuenca, 2012), el ocio se revela en la actualidad como un agente extraordinario de reestructuración del imaginario urbano y la producción del espacio público. Es llamativo constatar cómo la volatilización del espacio real bajo las oportunidades masivamente brindadas por la digitalización se ha visto acompañada, desde el ámbito del ocio, por una forma lúdica de experiencia urbana hiperreal. Por ejemplo, la que facilitaban simuladores de ingeniería social, como el clásico videojuego Simcity, en cuyo manual de usuario se podía leer:

Cuando juegas a Simcity, tú diseñas, construyes y gestionas ciudades. (...) Eres el responsable. Si tu ciudad es un agradable para vivir, tu población crecerá. Si no lo es, tus Sims dejarán la ciudad. Y estate seguro de que te harán saber lo que piensan de ti y tus políticas (...). Uno de los retos más duros de Simcity 2000 es mantener una enorme ciudad sin sacrificar la calidad de vida de tus Sims, sin arruinarte por mantener las infraestructuras y sin subir los impuestos tanto que los negocios se deslocalicen (Soja, 2008: 473).

El producto más evolucionado de esta saga de simuladores fue Simcity Societies, lanzado en 2007, en que el usuario se investía con los poderes, no ya de un gestor urbanista a escala municipal, sino con los de un primer ministro. Este género de sustituciones hiperrealistas de la realidad, por otros medios, han acabado poblando la ciudad de espacios simbólicos que simulan un tema en particular o un paquete de paisajes-imágenes. Autores como Edward Soja han abordado de manera muy perspicaz esta Disneyización o "parque-tematización" del espacio público urbano de lo que denomina la "postmetrópolis" contemporánea; espacios cuyas comunidades pueden elegir entre visitar "Fantasilandia, Aventurelandia, Fronteralandia, La Tierra del Mañana o El Pueblo de los Dibujos Animados" (Soja, 2008: 474), cada uno con su imaginario ad hoc y su colección de regulaciones formales e informales. 
Con todo, hay prácticas de ocio contemporáneas constitutivamente híbridas y con un carácter marcadamente juvenil -tanto por el perfil de sus practicantes como por su contribución al actual ideal de juventud (Cuenca, 2013)-, que ayudan a entender que cualquier metaforización de la ciudad (la ciudadescenario, tanto como la ciudad-lienzo o la ciudad-interfaz) tiene otras derivas y no todas son proclives a un grado de institucionalización como el descrito hasta aquí. La producción de un espacio público con una creciente tendencia a vaporizarse en nombre de la conectividad telemática, el Internet de las cosas (Smart-cities), la versatilidad funcional y la "eventualización" digna de su nuevo estatuto de escenario, no debe llevarnos al error de pasar por alto la cotidiana pugna por recuperar territorios de la ciudad mediante intervenciones y prácticas que basculan entre lo deportivo, lo cultural y lo lúdico. En este artículo seleccionaremos, precisamente, una práctica de ocio juvenil por cada uno de estos tres ámbitos: el street skateboarding, el graffiti y el juego de realidad aumentada Pokémon Go.

Se trata de tres prácticas muy heterogéneas en cuanto a origen, trayectoria, recursos, hábitos o destrezas, pero todas ellas tienen en común algunos rasgos que van a estar presentes en nuestro análisis. Interactúan con el espacio fáctico proyectando sobre él itinerarios que obedecen a reglas prácticas y necesidades recreativas ni previstas ni amparadas por las funciones originalmente asignadas al mismo. Son formas de reescritura del espacio, suponen formas de reterritorialización que, más que a la necesidad neotribal de visibilizar el control simbólico que una determinada subcultura ejerce en un determinado espacio urbano (Maffesoli, 1990), expresan alternativas imaginativas a la creciente incorporeidad y estandarización de nuestros paisajes metropolitanos, poniendo de relieve formas de leer y corresponsabilizarse con el entorno que, sin ser punibles, parecen marginales por estar situadas fuera del consenso funcional asumido por el ethos mayoritario de los ciudadanos. Son, en fin, marcas territoriales que reestructuran el hábitat urbano conforme a itinerarios de prácticas que rozan con los usos previstos y consensuados en torno a los espacios de las ciudades. A la luz del análisis de estas prácticas pretendemos ofrecer una respuesta a la cuestión por el papel de las formas contemporáneas del ocio juvenil en la reestructuración del imaginario urbano y la producción del espacio público en la actualidad.

\section{METODOLOGÍA}

Tras habernos detenido, con carácter general, en algunas de las dinámicas urbanas más recientes, que ofrecen contextos siempre polémicos a la experiencia de la ciudad, la metodología que empleamos pretende analizar cada una de las prácticas seleccionadas desde sus propias lógicas sociales internas, atendien- 
do a los lenguajes con que ellas imaginan, planean y se apropian de sus interactuaciones con el espacio público. Estos son algunas de las variables que pretendemos explorar transversalmente: primero, cómo prácticas que se constituyen en manifestaciones específicamente urbanas del deporte al aire libre, la creatividad plástica y la "gamificación", interactúan con un espacio entendido como producto social; segundo, cómo dichas interacciones contribuyen a imaginar reestructuraciones del ecosistema urbano, conforme a códigos de conducta y lenguaje de los que emergen nuevos sentidos de pertenencia a la ciudad, a la vez que re-significan el espacio público heredado; y, tercero, cómo esas prácticas -del deporte, la plástica o el videojuego "urbanizados"-, no sólo son proyectos alternativos de cartografía de la ciudad, sino que se constituyen en espacios (vividos) dentro del espacio (recibido), con lo que ayudan a definir funciones y oportunidades del territorio conforme a lógicas que están -o al menos nacen- en los márgenes de esa versatilidad de nuestras metrópolis hoy ya políticamente institucionalizada y que a menudo encuentran una respuesta de larvada o explícita hostilidad.

Tabla 1. Esquema de las categorías de interpretación

\begin{tabular}{l|l|l|l}
\hline Ámbito del ocio & Tipo de práctica & Metáforas de la ciudad & $\begin{array}{l}\text { Reestructuración } \\
\text { del imaginario urbano }\end{array}$ \\
\hline Deportivo & Skate & La ciudad-escenario & $\begin{array}{l}\text { Movimiento: destrezas } \\
\text { que vencen sobre los } \\
\text { usos previstos del } \\
\text { mobiliario urbano }\end{array}$ \\
\hline $\begin{array}{l}\text { Creativo } \\
\text { (plástico) }\end{array}$ & Grafitti & La ciudad-lienzo & $\begin{array}{l}\text { Lenguaje: la plasticidad } \\
\text { marginal sobre soportes } \\
\text { estructurales y de } \\
\text { delimitación }\end{array}$ \\
\hline Lúdico & Pokémon-Go & La ciudad-interfaz & $\begin{array}{l}\text { Logro: recorridos físicos } \\
\text { que se hibridan y } \\
\text { justifican mediante } \\
\text { motivos digitalmente } \\
\text { diseñados }\end{array}$ \\
\hline
\end{tabular}

\section{RESULTADOS Y DISCUSIÓN}

\section{Street skateboarding: el uso imprevisto del escenario urbano}

Surgido en el sur de California a lo largo de los años 50 como práctica de ocio juvenil, el skateboarding conoció su primera popularización a comienzos de la 
década siguiente, cuando la industria de tablas de surf comenzó a fabricar skates para su comercialización masiva (Beal, 2013; Borden, 1998a). En un primer momento, el skateboarding fue concebido por sus practicantes y comunicado públicamente como una extensión del surf en tierra firme, una forma de sidewalk surfing (surf de acera). En esta primera escena californiana, el stakeboarding comenzó a practicarse en espacios encontrados, especialmente propicios para la imitación de ciertas maniobras del surf (como piscinas vacías), y no fue sino hasta avanzados los años 70 cuando aparecieron los primeros skateparks, entornos construidos ad hoc para facilitar ejercicios de creciente dificultad acrobática (Borden, 1998a). A finales de los 80 surge propiamente el street skateboarding, que abandona las pistas de los skateparks para ocupar el centro mismo de las ciudades, aprovechando el mobiliario urbano para sus rutinas exhibitorias (Beal, 2013, pp. 19-21).

Como el resto de variedades, el street skateboarding se estructura alrededor de los trucos (tricks): un vasto repertorio de maniobras cuyo progresivo dominio va marcando la destreza del practicante. En lo que constituye todo un patrimonio comunitario de los skaters a nivel global, los trucos son movimientos cuidadosamente codificados, sometidos a unas exhaustivas nomenclatura (nollie, pop, FS pop, etc.) y tipología (trucos de trip, de grab, old school, etc.) y en ocasiones incluso vinculados al recuerdo colectivo de su inventor (como es el caso del ollie, creado por Alan "Ollie" Gelfand en los 70). Cada skater se ve obligado a repetir pacientemente un cierto número de movimientos hasta que domina el truco correspondiente. Aun cuando esto se ejecute en comunidad, a menudo por turnos y ante la mirada de otros practicantes, cada skater compite sobre todo consigo mismo; en un skate spot (lugar de reunión de skaters) se encontrará antes un acicate a la emulación que un verdadero sentido de competencia. Las actitudes patentemente competitivas son juzgadas con severidad: es el desarrollo de un estilo propio de autoexpresión creativa lo que se valora (Beal y Weidman, 2003).

La peculiaridad del street skateboarding consiste en que esta búsqueda colectiva de la destreza individual tiene lugar en el espacio público de la ciudad. Aun cuando cualquier paraje urbano podría concebirse como un skate spot en potencia, pueden identificarse una serie de características que concurren indefectiblemente en los lugares más exitosos entre los practicantes (Woolley y Johns, 2001). En primer lugar, el espacio debe ser fácilmente accesible desde otras partes de la ciudad, por lo que a menudo se localiza en ubicaciones céntricas, cercanas a nodos de la red viaria y del transporte público. En segundo lugar, la disposición del terreno y del mobiliario urbano debe ofrecer las condiciones adecuadas para el aprendizaje y la ejecución de trucos (por ejemplo, 
por medio de la presencia de escalones, barandillas o rampas). En tercer lugar, debe facilitar, al mismo tiempo, la sociabilidad entre practicantes, lo que a menudo implica que quienes están descansando puedan contemplar los ejercicios y exhibiciones de los demás desde los márgenes. Por último, los skaters suelen elegir espacios cuyo uso sea compatible con el tránsito de peatones, al menos en ciertos horarios (Woolley y Johns, 2001, pp. 214-215).

Esta última prevención no evita que el street skateboarding se perciba como más amenazador, en términos generales, que otras actividades deportivas en el espacio público, vistas bajo una luz más positiva (Monteagudo y Morejón, 2016), lo que parece deberse a su demanda de una acción física no estructurada de antemano, con altas dosis de improvisación y sin la subordinación a ninguna meta externa predeterminada (Beal y Weidman, 2003, p. 344). Son precisamente estas características de su práctica las que conducen a los skaters a desarrollar un peculiar imaginario en torno al espacio urbano que usan como territorio lúdico (Borden, 1998a, 2001a, 2001b; Woolley y Johns, 2001). Este uso sólo es posible porque arraiga en el espacio moderno descrito por Lefebvre: un espacio homogéneamente pavimentado, tendente al plano y la línea recta, cuya fragmentación en una serie de parcelas funcionales jerarquizadas favorece una notoria diversidad de elementos divisorios y de mobiliario urbano. A un tiempo, el street skateboarding surge de este tipo de entorno urbano y se rebela contra él, asignándole una serie de usos y significados imprevistos que subvierten las funciones oficialmente sancionadas y las someten a una "crítica performativa" (Borden, 2001b). Un elemento de seguridad, por ejemplo, como la barandilla de unas escaleras, se convierte para el skater en un estímulo para maniobras exhibitorias de alto riesgo. Este ejercicio de reasignación creativa de significados explica la potencia desestabilizadora del skateboarding:

The meaning of the skateboard move then in part takes its power and vitality from the fact that it comes out-of-the-blue, an unexpected and sudden eruption of meaning where society had previously been content to say nothing. Skateboarding is a critique of the emptiness of meaning (Borden, 2001b: 187).

Cabe ver en esta capacidad de resignificar el espacio cotidiano en entornos delimitados de acción lúdica un reverso de la "parque-tematización" de la que habla Soja (2008), aquí desligada de sus compromisos con los intereses hegemónicos de la postmetrópolis contemporánea. De hecho, esa vocación de subvertir los sentidos del espacio público ha llevado a que el street skateboarding sea percibido con frecuencia como una amenaza por los agentes administrativos y económicos de la ciudad, que no dudan en tomar medidas para impedir o al menos limitar su práctica en muchos centros metropolitanos alrededor 
del mundo y tratar de salvaguardar así la solidez de las funciones y significados atribuidos a la trama urbana (Németh, 2006). Tales medidas van desde la imposición de sanciones económicas cuantiosas hasta la intervención física sobre el espacio urbano mediante la adición de pinchos a las barandillas o la instalación de pavimentos rugosos o de gravilla (Borden, 1998b).

\section{Graffiti: la ciudad como lienzo inagotable}

La escena contemporánea del graffiti surge en el Nueva York de los años 70 en el marco de la cultura juvenil afroamericana del hip-hop, junto a otros elementos característicos como el rap o el breakdance. Frente a anteriores manifestaciones gráficas en los muros urbanos (tan antiguas como la ciudad misma), el graffiti se rige por unas reglas muy características por las cuales el practicante (writer, escritor), para ganarse el respeto de sus pares, debe lograr la máxima visibilidad en un entorno hostil a su trabajo y que siempre está dispuesto, por tanto, a eliminarlo del espacio público (Macdonald, 2001). Esto lleva a buscar la colocación de piezas muy elaboradas y de gran calidad estética en lugares especialmente visibles por su importancia funcional en la trama de tránsitos urbanos (como los vagones de metro, por ejemplo) o cuyo difícil acceso asegure la permanencia (como la estructura de un acueducto). Pero, al mismo tiempo, obliga a compensar esa tarea de elevado riesgo y alta inversión de tiempo y recursos con otra actividad menos comprometida: el tagging, es decir, la proliferación del propio tag (o firma) por toda la ciudad (Macdonald, 2001; Taylor, 2011). El tag de un escritor es un signo idiosincrático que debe poder realizarse rápidamente en cualquier lugar, a menudo con un rotulador grueso. Sólo desarrollando una marca reconocible por la comunidad del graffiti puede un escritor ir completando su proceso de "dejarse ver" (getting up) e ir creándose una reputación. La especificidad del graffiti como práctica de ocio juvenil se malinterpretaría si se concibiera centrada exclusivamente en el resultado estético de cada pieza: su dimensión territorial goza de igual importancia (si no superior).

Pese a que las prácticas visibilizadoras del control simbólico del entorno, identificadas por Maffesoli (1990) en su exploración del neotribalismo tardomoderno, constituyen el más amplio contexto de la dimensión espacial del graffiti, esta se ha hecho acreedora, frente a otras, de un tratamiento particularmente alarmista o condescendiente por parte de la literatura científica. Por un lado, desde los años 70 no han dejado de sucederse las interpretaciones que han procurado subsumir el fenómeno en los usos de marcado territorial entre grupos rivales de adolescentes potencialmente inclinados a una trayectoria delictiva (Ley y Cybriwsky, 1974; Taylor, 2011); por otro lado, se ha conceptua- 
lizado la búsqueda de visibilidad del escritor a través del tagging como una práctica adictiva y cercana a la psicopatología (Pani y Sagliaschi, 2009; Taylor, 2012). Halsey y Young (2006) argumentan que este tipo de lecturas, y otras equivalentes en términos de clase o género, dificultan cualquier aproximación honesta al modo en que los propios escritores viven y se explican su práctica, algo que hacen predominantemente en términos de placer y orgullo. La consideración de la práctica como fuente de placer, esencial para su comprensión como forma de ocio juvenil, debe comprenderse en toda su complejidad: hay una satisfacción emocional a la vista del desarrollo de destrezas específicas, una potente descarga de adrenalina derivada de la ilegalidad de la práctica y, sobre todo, una sensación de que se está haciendo "lo correcto" al intervenir sobre un muro (Halsey y Young, 2006). Esta última nota, quizá la más difícil de comprender desde fuera del mundo del graffiti, revela que los escritores experimentan el espacio urbano de un modo peculiar.

A uni-coloured wall is considered 'boring' -as 'negative space'- and therefore as something to be filled out or brought to life. (...) Most importantly, for many writers the surfaces that make up the built environment present more in the order of a flow than a structure. They are, in other words, canvasses permanently in waiting. (Halsey y Young, 2006: 288)

En términos generales, puede decirse que un escritor de graffiti imagina la ciudad como un espacio lleno de posibilidades para lograr el reconocimiento de sus pares a través de un ejercicio de auto-expresión creativa: la ciudad no aparece como un todo homogéneo organizado en diversas funciones jerarquizadas, sino como un plano continuo de múltiples superficies (Halsey y Young, 2006: 296). Los muros, que habitualmente cumplen una función delimitadora entre diversas formas y estatutos de propiedad del espacio, aquí se reinterpretan de un modo totalmente distinto. Frente a una concepción "ortogonal", por la cual el muro separa entre un adentro y un afuera, se instaura un uso "longitudinal" que conecta un aquí con un allí, como si cada pared fuera parte de una conversación vertical ilimitada entre pares (Brighenti, 2010: 329). Esta comprensión del espacio urbano implica una neutralización de todos los signos y marcas que lo ordenan habitualmente, una crítica-desde los márgenes- de las jerarquías de uso y función oficialmente sancionadas. Como afirma el antropólogo Néstor García Canclini:

Las luchas por el control del espacio se establecen a través de marcas propias y modificaciones de los graffiti de otros. Sus referencias sexuales, políticas o estéticas son maneras de enunciar el modo de vida y de pensamiento de un grupo que no dispone de circuitos comerciales, políticos o massmediáticos para expresarse, pero que a través del graffiti afirma su estilo. Su trazo manual, 
espontáneo, se opone estructuralmente a las leyendas políticas o publicitarias "bien" pintadas e impresas, y desafía esos lenguajes institucionalizados cuando los altera. El graffiti afirma el territorio pero desestructura las colecciones de bienes materiales y simbólicos. (Canclini, 2001: 306)

De ahí que la actividad de los escritores de graffiti sea percibida, no sólo como un acto de vandalismo por el público general, sino como una auténtica amenaza por los actores que quieren preservar el monopolio en el poder de marcado y ordenación del espacio urbano. Desde los años 70 hasta la actualidad, las autoridades de la ciudad de Nueva York, cuna de esta práctica de ocio juvenil, han llevado a cabo una auténtica cruzada contra el graffiti (Dickinson, 2008; Kramer, 2010), imitada por muchas otras ciudades en otras partes del mundo. En última instancia, este tipo de campañas anti-graffiti se revelan solidarias de una concepción de la ciudad que privatiza el espacio público y criminaliza cualquier intervención sobre el mismo que no tenga finalidad comercial (Dickinson, 2008).

\section{Pokémon Go: jugando en la interfaz físico-digital}

En julio de 2016, la compañía estadounidense de desarrollo de software Niantic lanzó Pokémon Go, un juego de realidad aumentada geolocalizada para dispositivos móviles iOS y Android. El juego aprovechaba buena parte de la tecnología aplicada en un proyecto anterior de Niantic, denominado Ingress, pero, gracias a un acuerdo de colaboración con Nintendo, sacaba partido a la franquicia Pokémon, un vasto conjunto de personajes ficticios que han protagonizado videojuegos y series televisivas de animación desde los años 90. Fue probablemente esta elección de contenidos la que convirtió el lanzamiento de Niantic en todo un éxito a nivel global, acercando al gran público, por primera vez, un proyecto que combinaba realidad aumentada y geolocalización. Aunque el fenómeno fue disminuyendo en intensidad a medida que quedaba atrás el verano de 2016, Pokémon Go fue una de las aplicaciones móviles más populares del año y en febrero de 2017 superaba ya los 650 millones de descargas (Sarkar, 2017). Las primeras estimaciones calculaban que el 70\% de los usuarios de la aplicación eran menores de 35 años, lo que la convierte en uno de las prácticas de ocio juvenil de los últimos años con mayor impacto a nivel global (YouGov, 2016).

El objetivo del juego consiste en capturar todos los Pokémon posibles y hacerlos evolucionar. Para conseguirlo, el jugador debe desplazarse físicamente por su entorno cercano, el cual visualiza en forma de mapa en su dispositivo móvil. Cuando se encuentra en la cercanía de un Pokémon, puede cambiar a la vista de realidad aumentada, en la que el personaje digital se le aparece superpuesto a la imagen del entorno físico, captada por la cámara del disposi- 
tivo móvil. Determinados puntos geolocalizados son relevantes para el avance en el juego: las Poképaradas, donde el usuario puede encontrar objetos necesarios para capturar y hacer evolucionar Pokémon, y los gimnasios, donde los jugadores pueden enfrentar a sus Pokémon entre sí. Ambos tipos de ubicaciones están localizadas, por lo general, en puntos de interés del espacio público, tales como monumentos, parques o fuentes, aunque a lo largo de 2016 compañías como McDonald's o Starbucks también llegaron a acuerdos para colocar Poképaradas y gimnasios en algunos de sus locales (Mochizuki, 2016; Starbucks, 2016). Desde el comienzo, el tratamiento del fenómeno que hicieron los medios de comunicación se centró principalmente en la ubicación -en ocasiones polémica- de estos puntos geolocalizados y en los diversos problemas ocasionados por la afluencia de usuarios del juego a los mismos (que en ciertos casos llegó a ser ciertamente masiva).

Buena parte de la creciente literatura científica sobre Pokémon Go se ocupa también de sus posibles impactos negativos y riesgos asociados. Tan pronto como en agosto de 2016, un artículo publicado en PARIPEX. Indian Journal of Research, exponía las fases que podían conducir al jugador a un comportamiento compulsivo y concluía con estas alarmantes palabras: "Before the 'Pokemania' or 'Pokemon mania' drives individual crazy the sooner the consorted action is taken is the better" (Bhattacharyya, Ray, Bhattacharya y Mallick, 2016). Pero más allá de la jugabilidad, es la condición geolocalizada de la aplicación y, por tanto, el necesario tránsito de sus usuarios por el espacio urbano, lo que ha centrado la mayoría de los análisis. Así, por ejemplo, se ha alertado del riesgo de posibles traumatismos debidos a ceguera por falta de atención (Barbieri et al., 2017), así como del aumento de exposición de los jugadores a las enfermedades transmitidas por mosquitos (Oidtman et al., 2016). Por otro lado, también se han detectado posibles beneficios derivados del uso extensivo del espacio urbano al que se ven motivados los jugadores más comprometidos: se ha mostrado la influencia de Pokémon Go en el aumento de los niveles de actividad física (Alhoff, White y Horvitz, 2016; Nigg, Mateo y An, 2017; Wong, 2017) y se han señalado también sus posibles aportaciones a la conciencia de la protección de espacios naturales (Dorward, Mittermeier, Sandbrook y Spooner, 2017).

Con todo, no son numerosos los estudios que se ocupan en profundidad de la naturaleza híbrida del juego y analizan la peculiar experiencia del espacio urbano que facilita en el jugador la superposición entre el entorno físico y el digital. Como señalan Hjorth y Richardson (2017), la adición de elementos digitales permite que entornos banales y familiares se transformen en lugares de juego significativos. Es aquí donde reside la especificidad de Pokémon Go, 
que no ofrece un mero entretenimiento casual en los intervalos entre las ocupaciones cotidianas, sino que, más bien, interviene explícitamente sobre esas ocupaciones y rutinas y puede llegar a modificarlas (Hjorth y Richardson, 2017: 5). En efecto, un análisis geoestadístico a gran escala de diversos elementos del juego ha comprobado que Pokémon Go induce cambios en la movilidad de los jugadores. Estos parecen adaptar sus rutinas diarias para desplazarse a lugares cercanos a su domicilio o su lugar de trabajo donde puedan encontrar Pokémon y, además, tienden a moverse por la ciudad en pares o pequeños grupos con mayor frecuencia (Colley et al., 2017). Pero la naturaleza híbrida del juego no afecta sólo a las acciones de los jugadores en el espacio urbano, sino a su percepción, experiencia e imaginación del mismo. Dada la identidad que el juego presupone entre los movimientos del jugador por su entorno físico y los de su avatar en el espacio digital, no puede restringirse el significado del mapa mostrado en la pantalla del dispositivo móvil a un mero dispositivo representacional de orientación: por el contrario, debe entenderse como una compleja interfaz procesual que permite la navegación por una realidad híbrida (digital y analógica a un tiempo) -tal y como han mostrado Lammes y Wilmott (2011) para otros juegos basados en geolocalización-. Los puntos de interés del espacio digital se sobrescriben a los del entorno físico, y no se hacen notar con menor eficacia, ya sea a través de estímulos ópticos (realidad aumentada) o táctiles (el dispositivo Pokémon Go Plus, que consiste en una pulsera inteligente que vibra cuando un Pokémon se encuentra cerca). La peculiar condición híbrida de esta práctica de ocio juvenil la hace particularmente solidaria de las más recientes metáforas descorporeizadas del espacio urbano que, como señala Sloterdijk (2014), acaban subsumiendo la ciudad en una especie de imaginada macro-espuma de conexiones y tránsitos.

No obstante, el potencial de distorsión de las interacciones en el espacio público que acompaña a este tipo de experiencia territorial híbrida no debería desdeñarse. A lo largo del verano de 2016 se reportaron numerosos casos de comportamientos colectivos anómalos -como súbitas concentraciones de jugadores en busca de un Pokémon poco común (Chen, 2016)-, así como conductas individuales que se consideraron ofensivas -como la prosecución del juego en espacios muy cargados históricamente o con relevancia religiosa (Sarabia, 2016)-. En muchas ciudades del mundo, las autoridades no tardaron en tomar medidas prohibiendo el juego en áreas determinadas.

\section{CONCLUSIONES}

El skater que surfea por la trama material de la ciudad con un deslizamiento exhibitorio evadido de las necesidades de circulación, el escritor de graffiti que 
emplea la superficie de un edificio como bastidor de un lenguaje encriptado, el jugador que caza Pokémon virtuales en un entorno físico desde la interfaz de su smartphone: todos ellos reestructuran con sus prácticas el imaginario urbano, produciendo un espacio que es capaz de trascender algunos de los más fundamentales ordenamientos del urbanismo moderno, como son las zonificaciones funcionales y los estatutos de propiedad del suelo. Todos ellos "leen" de manera diversa el espacio. Crean una legibilidad alternativa de la ciudad. La legibilidad es de una importancia decisiva en el escenario urbano (Lynch, 2010:11): y esa legibilidad heterodoxa se produce en los bordes sobre los que se aplican preferentemente los skaters, cabalgando sobre los límites entre dos zonas de clases diferentes; en los nodos, focos estratégicos de la imagen de la ciudad, las confluencias que obligan al viandante a convertirse necesariamente en observador y a aguzar su atención, y que se convierten en la ubicación prominente del grafiti; o en los hitos, los puntos u objetos de referencia que se consideran exteriores a las personas y a los que casi todas las ciudades adhieren una historia para que así se refuerce su valor como mojón, hoy reinventados y reprogramados por Pokémon Go. Siguiendo los elementos constitutivos de la imagen de la ciudad estudiados en su día por Kevin Lynch, podría concluirse que, efectivamente, estas tres prácticas son otras tantas constelaciones desde las que se está reimaginando la ciudad. No hay en esto, quizá, como hemos tratado de mostrar, una diferencia que los oponga radicalmente a las últimas derivas en la metaforización de la ciudad contemporánea, cuyas instancias hegemónicas obstinadamente imaginan como dotada de infinita versatilidad, constituida por eventos y flujos, casi incorpórea. Ahora bien, el skater, el escritor de graffiti y el jugador de Pokémon Go no producen metáforas ad hoc desde el abstracto territorio del city branding, sino que ponen en práctica su imaginario urbano en una experiencia concreta, corpórea, incardinada en la trama viva de la ciudad. Es este anclaje el que convierte a estas tres formas del ocio juvenil contemporáneo en otras tantas estrategias posibles para recuperar y repoblar creativamente el espacio público de las ciudades tardomodernas.

Sería ingenuo, en cualquier caso, rodear estas prácticas de un aura utópica que prometiera un espacio urbano libre de contradicciones e inequidades. Se ha denunciado, por ejemplo, cómo el graffiti y el skateboarding canalizan formas de construcción de la masculinidad que invisibilizan el talento y la iniciativa de las mujeres participantes (Beal y Weidman, 2003; Macdonald, 2001), o cómo la distribución de Poképaradas refuerza las desigualdades entre vecindarios de diversa composición étnica (Colley et al., 2017). Tampoco puede ignorarse el origen corporativo de Pokémon Go que, frente a las otras prácti- 
cas, constituye en realidad un producto diseñado y distribuido por una compañía con afán de lucro. Con todo, estas consideraciones no pueden servir para anular la experiencia de los participantes ni para negar cualquier virtualidad a su impacto sobre los imaginarios urbanos. Aun en grados muy diversos, que van desde la persecución legislativa a la ridiculización mediática, estas tres prácticas de ocio juvenil han encontrado en el público general una reacción de mayor o menor hostilidad. Como afirma Dickinson (2008: 40) en referencia al graffiti, lo que anida en el fondo de estas polémicas es la cuestión sobre quién está autorizado a iniciar proyectos en el espacio público. Y a reimaginarlo por completo, añadimos.

\section{BIBIOGRAFÍA}

AA.VV. (2006). Urbanismo situacionista. Barcelona: Gustavo Gili.

Althoff, T., White, R.W. y Horvitz, E. (2016). Influence of Pokémon Go on Physical Activity: Study and Implications. Journal of Medical Internet Research, 18(12):e315, doi:10.2196/jmir.6759

Andreotti, L. y Costa, X. (Eds.). (1996). Teoría de la deriva y otros textos situacionistas sobre la ciudad. Barcelona: MACBA/Actar.

Aragon, L. (2016). El aldeano de París. Madrid: Errata Naturae.

Barbieri, S., Vettore, G., Pietrantonio, V., Snenghi, R., Tredese, A., Bergamini, M., Previato, S., Stefanati, A., Gaudio, R.M., Feltracco, P. (2017). Pedestrian Inattention Blindness While Playing Pokémon Go as an Emerging Health-Risk Behavior: A Case Report. Journal of Medical Internet Research, 19(4):e86. doi:10.2196/jmir.6596

Bayón, F. (2012). Las retóricas del público. El espacio de consumo del arte como institución política. Arbor. Ciencia, Pensamiento y Cultura, 188 (754), 409-426.

Beal, B. (2013). Skateboarding. The Ultimate Guide. Santa Barbara, CA: Greenwood.

Beal, B. y Weidman, L. (2003). Authenticity in the Skateboarding World. En R.E. Rinehart y S. Sydnor (Eds.), To the Extreme: Alternative Sports Inside and Out (pp. 337-352). Albany, NY: State University of New York Press.

Benjamin, W. (2005). Libro de los pasajes. Madrid: Akal.

Bhattacharyya, R., Ray, U., Bhattacharya, J., y Mallick, B. (2016). Pokemania, Threats and Concerns of A Simple Augmented Reality Game. PARIPEX-Indian Journal of Research, 5(8). 363-366.

Blumenberg, H. (1995). Naufragio con espectador. Paradigma de una metáfora de la existencia. Madrid: Visor.

Borden, I. (1998a). Body architecture: skateboarding and the creation of super-architectural space. En J. Hill (Ed.), Occupying Architecture: Between the Architect and the User (pp. 111-125). London: Routledge.

Borden, I. (1998b). An affirmation of urban life: Skateboarding and socio-spatial censorship in the late twentieth century city. Archis, 5, 46-51. 
Borden, I. (2001a). Skateboarding, Space and the City: Architecture and the Body. Oxford: Berg.

Borden, I. (2001b). Another Pavement, Another Beach: Skateboarding and the Performative Critique of Architecture. En I. Borden, J. Rendell, J. Kerr y A. Pivaro (Eds.), The Unknown City. Contesting Architecture and Social Space (pp. 178-199). Cambridge, MA / London: MIT Press.

Brighenti, A.M. (2010). At the Wall: Graffiti Writers, Urban Territoriality, and the Public Domain. Space and Culture. 13(3), 315-332.

Careri, F. (2013). Walkscapes. El andar como práctica estética. Barcelona: Gustavo Gili.

Chen, J. (16 de julio de 2016) Pokemon Go: Crowd Stampedes Through Central Park After Rare Vaporeon Sighting. US Magazine. Recuperado de http://www.usmaga zine.com/celebrity-news/news/pokemon-go-crowd-stampedes-after-spottingrare-pokemon-w429525

Colley, A., Thebault-Spieker, J., Lin, A. Y., Degraen, D., Fischman, B., Häkkilä, J. y Wenig, D. (2017). The Geography of Pokémon GO: Beneficial and Problematic Effects on Places and Movement. In Proceedings of the SIGCHI Conference on Human Factors in Computing Systems. doi:10.1145/3025453.3025495

Cuenca, J. (2012). Génesis de la comprensión experiencial del ocio en la modernidad tardía: transformaciones del concepto de vivencia. Arbor. Ciencia, Pensamiento y Cultura, 188(754), 315-325, doi:10.3989/arbor.2012.754n2005

Cuenca, J. (2013). Peter Pan disecado. Mutaciones políticas de la edad. Bilbao: consonni.

Debord, G. (2004). Introducción a una crítica de la geografía urbana. A Parte Rei: revista de filosofía, 11, 10-13.

Debord, G. (2010).Teoría de la deriva. Revista Anthropos: Huellas del conocimiento, 229, 197-200.

Dickinson, M. (2008). The Making of Space, Race and Place New York City's War on Graffiti, 1970 - the Present. Critique of Anthropology, 28(1), 27-45.

Dorward, L. J., Mittermeier, J. C., Sandbrook, C. y Spooner, F. (2017). Pokémon Go: Benefits, Costs, and Lessons for the Conservation Movement. Conservation Letters, 10, 160-165. doi:10.1111/conl.12326

Fargue, L.P. (2014). El peatón de París. Madrid: Errata Naturae.

García Canclini, N. (2001). Identidades híbridas. Estrategias para entrar y salir de la modernidad. Barcelona: Paidós.

Halsey, M. y Young, A. (2016). Our desires are ungovernable. Theoretical Criminology, 10(3), 275-306.

Hjorth, L., y Richardson, I. (2017). Pokémon GO: Mobile media play, place-making, and the digital wayfarer. Mobile Media y Communication 5(1), 3-14, doi: 10.1177/ 2050157916680015

Kramer, R. (2010). Moral panics and urban growth machines: official reactions to graffiti in New York City, 1990-2005. Qualitative Sociology, 33(3), 297-311.

Lefebvre, H. (2013). La producción del espacio. Madrid: Capitán Swing.

Ley, D. and Cybriwsky, R. (1974). Urban graffiti as territorial markers. Annals of the Association of American Geographers, 64(4), 491-505. 
Macdonald, N. (2001). The Graffiti Subculture: Youth, Masculinity and Identity in London and New York. Hampshire, UK / New York, NY: Palgrave Macmillan.

Maffesoli, M. (1990). El tiempo de las tribus. Barcelona: Icaria.

Mochizuki, T. (19 de julio de 2016). McDonald's Unit to Sponsor Pokémon Go in Japan. Wall Street Journal. Recuperado de https://www.wsj.com/amp/articles/ mcdonalds-unit-to-sponsor-pokemon-go-in-japan-1468936459

Monteagudo Sánchez, M. J., y Morejón Torné, S. (2016). Ocio deportivo en los waterfronts de Bilbao y Barcelona. Un estudio comparativo. Revista de Psicología del Deporte, 25(supl. 2), 15-20.

Nigg, C.R., Mateo, D.J., y An, J. (2017). Pokémon GO May Increase Physical Activity and Decrease Sedentary Behaviors. American Journal of Public Health, 107(1), 3738, doi:10.2105/AJPH.2016.303532

Oidtman, R.J., Christofferson, R.C., ten Bosch, Q.A., Espana, G., Kraemer, M.U.G., Tatem, A., Barker, Ch.M. y Perkins, T.A. (2016). Pokémon Go and Exposure to Mosquito-Borne Diseases: How Not to Catch 'Em All. PLoS Currents, 8, doi: 10.1371 /currents.outbreaks.2d885b05c7e06a9f72e4656d56b043cd

Pani, R. y Sagliaschi, S. (2009). Psychopathology of Excitatory and Compulsive Aspects of Vandalistic Graffiti. Psychological Reports, 105(3_suppl), 1027-1038.

Sarabia, D. (6 de septiembre de 2016). Un youtuber detenido en Rusia por jugar a Pokémon Go en una iglesia. Eldiario.es. Recuperado de http://www.eldiario.es/cul tura/tecnologia/Entrenador-juegues-Pokemon-Go-iglesia_0_556094518.html

Sarkar, S. (27 de febrero de 2017). Pokémon Go hits 650 million downloads. Polygon. Recuperado de https://www.polygon.com/2017/2/27/14753570/pokemon-godownloads-650-million

Sloterdijk, P. (2014). Esferas III: Espumas. Madrid: Siruela.

Soja, E.W. (2008). Postmetrópolis. Estudios críticos sobre las ciudades y las regiones. Madrid: Traficantes de sueños.

Starbucks (8 de diciembre de 2016). "Discover the Pokémon GO Frappuccino at Starbucks". Starbucks.com. Recuperado de https://news.starbucks.com/news/starbuckspokemon-go

Taylor, M. F. (2012). Addicted to the Risk, Recognition and Respect that the Graffiti Lifestyle Provides: Towards an Understanding of the Reasons for Graffiti Engagement. International Journal of Mental Health and Addiction, 10(1), 54-68.

Taylor, M.F. (2011). Hanging with the Hoodies: Towards an Understanding of the Territorial Tagging Practices of Prolific Graffiti Writers Seeking an Adolescent Nonconforming Social Identity. International Journal of Child and Adolescent Health, 4(3), 223-232.

Wong, F. Y. (2017). Influence of Pokémon Go on physical activity levels of university players: a cross-sectional study. International Journal of Health Geographics, 16(1), 8.

Wooley, H. y Johns, R. (2001). Skateboarding: The City as a Playground. Journal of Urban Design, 6(2), 211-230. 
YouGov (2016). Pokémon Go: 34m downloads, 31m current players, and 10m paying users since U.S. launch. YouGov.com. Recuperado de https://today.yougov.com/news/ 2016/08/12/pokemon-go-34m-downloads-31m-current-players-and-1/

FERNANDO BAYÓN (Bilbao, 1971) es Doctor en Filosofía por la Universidad de Deusto (UD). Actualmente, es Investigador de la Línea "Ocio y desarrollo humano" del Instituto de Estudios de Ocio de la Facultad de Ciencias Sociales y Humanas de UD, en la que es también profesor de "Historia de Filosofía en la Modernidad: Idealismo y Dialéctica", dentro del grado de "Filosofía, Política y Economía". Es autor y/o editor de más de cincuenta publicaciones científicas dedicadas a la construcción narrativa de la memoria en el espacio político europeo. Entre sus libros, se cuenta: "Filosofía y Leyenda: Variaciones sobre la última modernidad (de Tolstói a Musil)" (Barcelona, Antrhopos, 2009). orcid.org/0000-0002-4465-8643

JAIME CUENCA (Bilbao, 1983) es Doctor en Ciencias Sociales y Humanas y licenciado en Filosofía. Actualmente, es investigador asociado en el Instituto de Estudios de Ocio de la Universidad de Deusto y miembro del equipo de investigación reconocido Ocio y Desarrollo Humano. Ha impartido docencia en la Universidad de Deusto y en el Tecnológico de Monterrey en el ámbito de la teoría del ocio, estética, filosofía del arte y otras disciplinas filosóficas. Es autor de dos monografías y más de una veintena de artículos en revistas especializadas, tales como Arbor, World Leisure, Pensamiento, Journal of Leisure Research o Estudios filosóficos. Ha contribuido a varios catálogos de artista y ejerce como crítico de arte en diversos medios de prensa escrita.

JOSÉ ANTONIO CARIDE es Catedrático de Pedagogía Social en la Facultad de Ciencias de la Educación de la Universidad de Santiago de Compostela, en la que es el Comisionado de su "Campus da Cidadanía" en las áreas de Ciencias Sociales y Jurídicas, Artes y Humanidades. Dirige el Grupo de Investigación "Pedagogía Social y Educación Ambiental" (SEPA-interea). Presidió la Sociedad Iberoamericana de Pedagogía Social (SIPS), entre 2002 y 2013. En 2004 le fue concedida la "Orden al Mérito Institucional" del World Council For Curriculum and Instruction. Sus publicaciones y líneas de investigación pueden consultarse en: https://usc-es.academia.edu/Jos\%C3\%A9AntonioCaride. Código orcid: orcid.org/0000-0002-8651-4859 